\title{
Comparative Study of Deep Learning Methods in Detection Face Mask Utilization
}

\author{
Ivan Muhammad Siegfried ${ }^{1}$ \\ ${ }^{1}$ Independent Researcher \\ *Corresponding author's email: ivanmuhsiegfried@gmail.com
}

\begin{abstract}
A BS T RA C T
In 2020, the world is facing new and emerging virus called COVID-19 where the transmission could be halted using a face mask. A method and model needed to anticipate the spread of such virus. We study some transfer and deep learning methods: MobileNetV2, ResNet50V2, and Xception. The result is that the usage of ResNet50V2 and Xception for face image dataset using mask has better accuracy and precision than that of MobileNetV2 method.
\end{abstract}

Keywords: COVID-19, Deep Learning, Detection, Face, Mask

\section{Introduction}

In 2020, the utilization of a face mask has been massive because mankind faces an emerging pandemic caused by SaRS-COVID-19. The use is a preventive step of COVID-19 spread[1]. COVID-19 is spread because of droplet that thrown by cough and sneeze. Although it was debated, the study found that there is a tendency for the COVID-19 virus to transmit either through droplets or through the air for three hours after the aerosolization process [2]. These facts further prove that the utilization of masks as a means of protecting humans facing this pandemic is getting bigger and bigger.

Many people are now competing to use a lot of tools to overcome the related problem with this pandemic. In Internet-of-Things (IoT) field, identification and monitoring of early COVID-19 symptoms between residents and health authorities was proposed[3]. Then, there is also drone development that minimizes direct contact between patients and health workers in a hospital[4]. Another example is that in the field of machine learning, calculations and forecasts regarding to epidemic growth based on datasets taken from the government authorities and the public data was published[5].

There are several strategies in which people using face mask in certain place and case[6]. The utilization of mask by people living in areas where there is no social gathering and proper ventilation is considered unnecessary to use masks. However, some specific places such as shopping places, offices, restaurants, etc. and the rooms having closed ventilation are considered to have varying risks from high to low as a place where there is risk of transmitting the COVID-19 virus. In addition, workers who are susceptible to have virus exposure need to wear mask from low to high level.

The use of masks in society is still a challenge. According to writer's observation, In Indonesia, for example, there are residents who are reluctant to use masks. Several reasons are the people's health condition, misinformation and misinterpretation, politics, beliefs, mental health conditions and herd immunity. Another thing that becomes a challenge is that people are getting tired of using masks. Another challenge is the limited authorities' personnel which resulted the monitoring of masks usage becomes less and less effective.

To overcome such problem of ineffective monitoring, this paper proposes a method to detect face mask through image that can be produced by cameras or image files. To detect, we use and compare the method 
using classification method called as MobileNetV2, ResNet50V2, and Xception in deciding whether a face image wears a mask or not.

In this study, we compared several pre-trained artificial neural methods in comparing the accuracy of each method suitable for detecting the use of mask. We will start with the systematics of writing by introducing the dataset and methods used in the augmentation process to increase the information from the image to be studied. Then, we will discuss about the transfer learning method using the MobileNetV2 algorithm, compared with ResNet50V2, and Xception to be combined with facial image recognition model called Caffe Model. All in all, we will discuss the results and discuss the performance of each transfer learning method as well as program implementation.

\section{Dataset and Methods}

Data set used in this research is the one Chandrika gathered [7] which having crawled from Bing Search API, Kaggle Dataset, and Real World Masked Face Dataset[8]. This dataset has variability of background image resulting in the time needed to be converged is longer.

\subsection{Pre-processing: Augmentation}

Pre-processing is a first step in improving image information such as elimination distortion so that it can be used to process information better. In this study, we use several types of pre-processing, namely augmentation, which aims to avoid overfitting so that if the device faced with certain problem with micro differences encountered, the program can still make predictions correctly.

Augmentation is a process of increasing variability using training data sets. The training data is then transformed randomly involving random rotation, image resizing, shifting, and so on. The augmentation process in Tensorflow itself replaces the training data with new training data that has been transformed according to the wishes of the users themselves. The augmentation process itself in Tensorflow does not add to training data but transforms it.

There are several augmentation parameters that have been added in this program: rotation, flip, and image shift from the original point (range shift). These augmentation parameters are needed because the variations in image capture using the camera are quite varied: face tilt, camera flip direction, and images that move at a position not at a certain point.

\subsection{Image Classification Using MobileNetV2, ResNet50V2, and Xception}

One of the architectures suitable for machines that have low power is MobileNets. MobileNets are one type of neural network architecture which is the development from the first generation, namely MobileNetV1. MobileNetV2 uses two blocks: residual and downsizing. For the two block types, there are three layers where the first is the $1 \times 1$ convolution using ReLU6 using the activation function, the second layer uses deep convolution and uses ReLU, and the last one is the $1 \mathrm{x} 1$ convolution layer using a linear activation function. When compared to MobileNetV1 and ShuffleNet 1.5, the use of MobileNetV2 has a better performance in terms of the computational load and has an effect on the shorter iteration time[9].

MobileNetV2 is an architecture for describing neural networks using a pre-trained model. This pre-trained model is a training model in the form of an artificial neural network whose parameters are already owned and ready for adding layers or what is commonly called fine-tuning. In carrying out the training process, 
the artificial neural network layers that are owned by MobileNetV2 are not subjected to value updating, but a fine-tuning layer is carried out by updating the values according to what is defined by the user.

In addition, the Deep Convolutional Neural Network (CNN) method that will be used next is ResNet50V2[10]. This method is an extension of the ResNet50 method which runs better than its predecessor. Some of the breakthroughs made by ResNet are by involving back propagation training to help minimize the loss function to quickly get a solution for the neural network model. one of the main differences between Resnet and ResNetV2 is the use of batch normalization for each weight layer.

Meanwhile, the DCNN Method we will use is the Xception Method. This method consists of several flows: entry flow, middle flow, and exit flow. When compared to ResNet50 which has a layer depth of 50, Xception has a depth of 71 layers. This method can classify objects up to 1000 categories.

\subsection{Face Recognition Using Caffe Model}

Caffe Model is used to get features of image. These features are obtained using previously trained training. Some of the abilities used include object classification, studying the semantic features of an image, and object detection. Caffe provides a reference model for solving visual problems.

In this study, the Caffe Model is used to perform a face selection in the image produced by the input device. This needs to be done so that the prediction process that has been carried out at the training data stage produces the expected value. To use Caffe Model, it is necessary to use several files that OpenCV requires: a file with the extension .prototxt containing the neural network configuration, and the file with the * .caffemodel which contains the weight values of the previously trained model.

\subsection{Proposed Methods}

Carrying out the training process, we used a Toshiba P55W portable computer using an i5-5200U @ $2.20 \mathrm{GHz}$ processor (4 CPU) without using a GPU. Some of the syntax is inspired by the Face Mask Detection program created by Chandrika[10]. The interpreter we use is python with version 3.7 with Anaconda 3 as a Virtual Environment. The amount of division between training data and validation data is $80: 20$. This validation data is needed to determine the system performance against new data that is not included in the training data. Some of the parameters used in this study are:

- The augmentation used is a rotation distance of 20 , zooming area in the range of 0.15 , side and top shift of 0.2 , and image shift of 0.15 .

- The batch_size used is 15

- The basic artificial neural network models that were tested are MobileNetV2, ResNet50V2, and Xception to see how the accuracy value is.

- The top model used these layer: AveragePooling2D with a pool_size of (7.7), then continued with Flatten, then proceed to the Dense layer with a size of 128 which has the ReLU activation function, then the arrays are dropped by 0.5 and continued to the Dense layer of 2 which uses the SoftMax activation function. The Dense value of 2 is adjusted to the output we want as many as 2 , namely the detection of using masks or not using masks.

- In the fitting process, the batch_size used is 15 , the steps per epoch are determined as the amount of training data divided by batch size. Then, in this fitting process, we define validation data where the number of validation steps is equal to the number of validation data divided by the number of batch sizes that we define. 
- After training model is completed, the model is stored in a file so that it can be easily used to carry out the task of classifying images from cameras, images, and video images.

- The face detection image uses the Caffe Model. The detected face is classified using a model that has been fine-tuned to see whether there is a mask or not on the face image.

\section{Results and Discussion}

\subsection{Image Training Process using MobileNetV2}

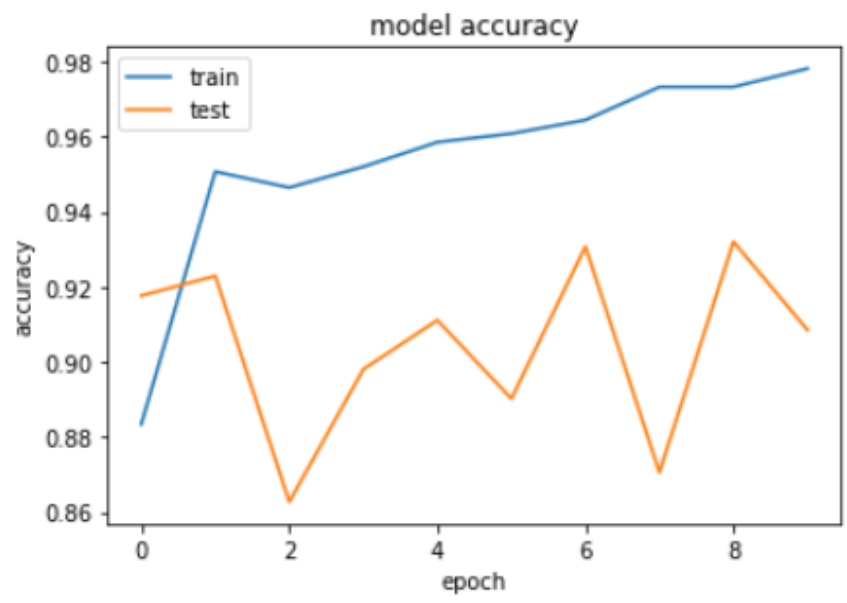

Figure 3-1: Image of Accuracy vs Epoch using Base Model MobileNetV2

In this training process, when the accuracy of the training data increases from the first epoch to the last epoch, the accuracy of the test data varies in the range 0.86 to 0.93 . This indicates that the use of the MobileNetV2 model is overfitting and carried out carefully. For a typical form of fluctuating data accuracy using this method, it is recommended that the user stop the training process by using the callbacks function. This callbacks function is an iteration termination function that is called when a training process has reached a certain level of accuracy. So, it is expected that the model used has the level of accuracy desired by the user. The time needed to run 10 epochs is $6926.81 \mathrm{~s}$.

\subsection{Image Training Process using ResNet50V2}

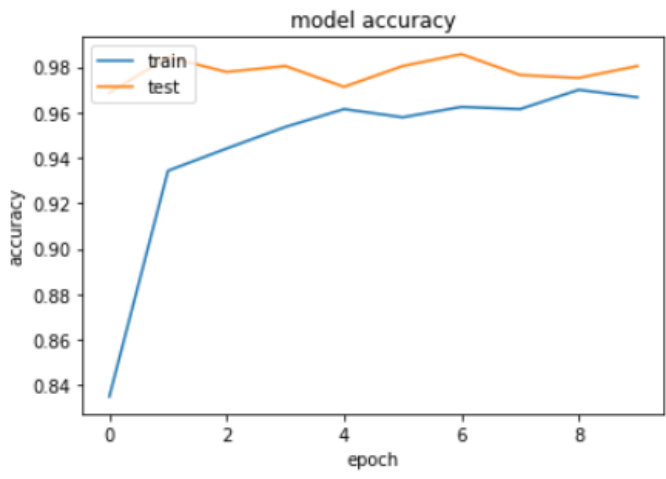

(a)

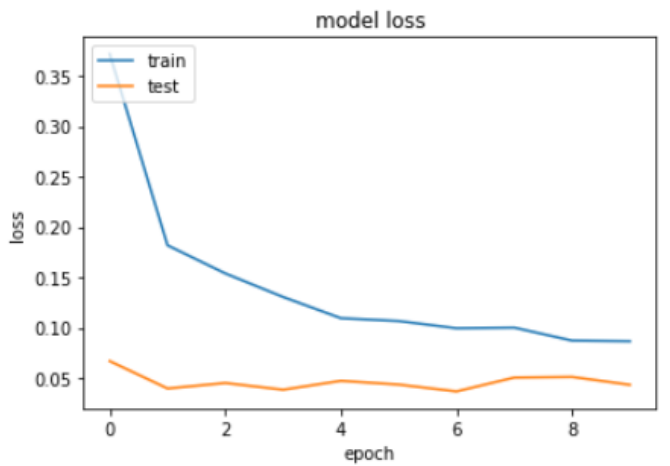

(b)

Figure 3-2: (a) Figure of Accuracy vs Epoch; and (b) Figure of Loss vs Epoch of ResNet50V2 Method

Different from MobileNetV2, the accuracy using ResNet50V2 for training data tends to be low at 0-th epoch. Even so, the accuracy model from the test data or what we usually call validation data moves in a relatively narrow range between 0.97 to 0.99 which is consistent from $0^{\text {th }}$ epoch to $9^{\text {th }}$ epoch. 
The loss value obtained for training data decreases. This indicates that the more training data (indicated by the number of epochs), the training data value will be closer to the original value (marked by a low loss value). Meanwhile, the loss value of the model using validation data is seen in the range 0.03 to 0.05 .

\subsection{Image Training Process using Xception}

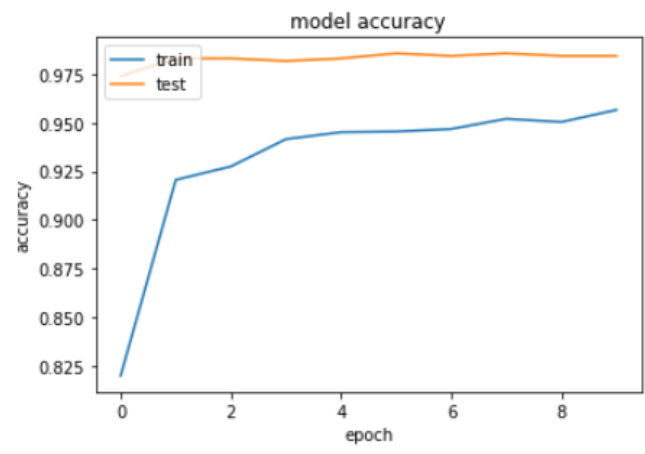

(a)

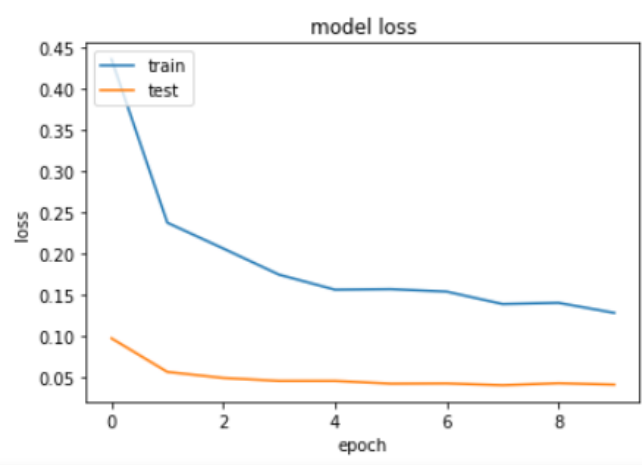

(b)

Figure 3-3: (a) Figure of Accuracy vs Epoch; and (b) Figure of Loss vs Epoch of Xception Method

Of the three methods used, the Xception method has the highest level of stability. This is because the accuracy model obtained for image classification using data validation (test) has a precise range in the range of 0.975 to 0.98 . However, similar to the modeling process using ResNet50V2, the use of the Xception method for training data tends to be lower. This indicates that the Xception and ResNet50V2 models are ideal because there is no overfitting as indicated by the low accuracy of the MobileNetV2 train dataset.

Table 1: Iteration Time of Pre-trained Deep Learning Methods Used

\begin{tabular}{|c|c|}
\hline Method & Iteration Time (s) \\
\hline MobileNetV2 & 6926.81 \\
\hline ResNet50V2 & 12551.99 \\
\hline Xception & 16232.06 \\
\hline
\end{tabular}

In general, if one has low resources but has a lot of training data and aims to train the data as accurately as possible, the use of Xception and ResNet50V2 methods is recommended for two to three times of epochs only. Although the iteration time using MobileNetV2 tends to be faster, in this case, overfitting is unavoidable so it is not recommended for mask detection in this case. 


\subsection{Performance of Mask Detection Model}

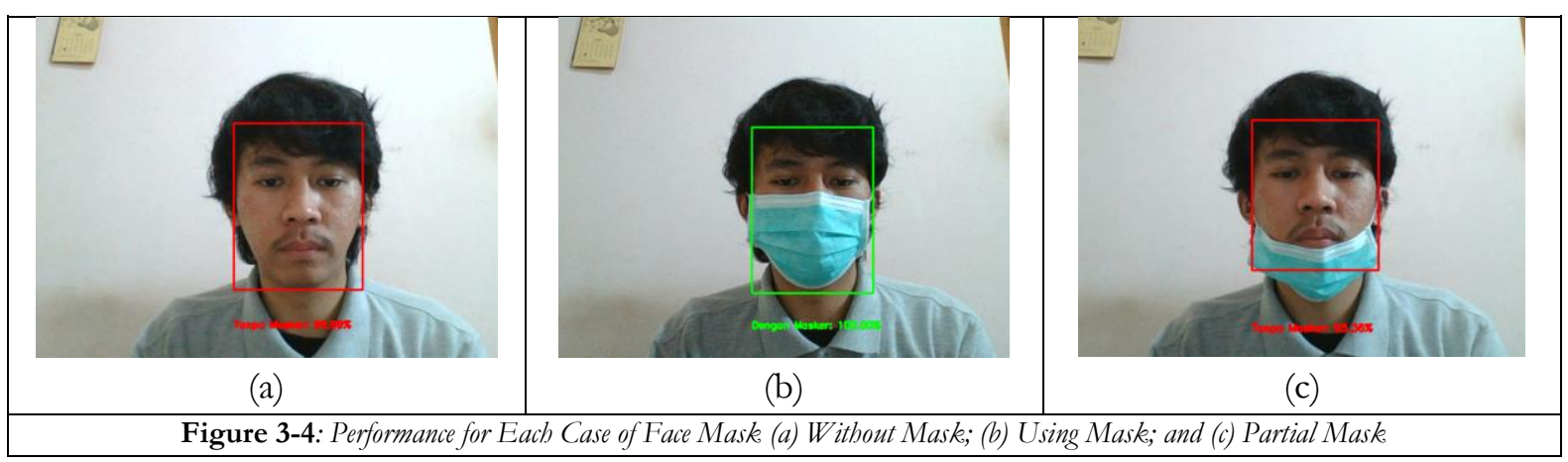

When it comes to the performance of the model, the system can detect facial images without a mask, with a mask, and partial mask (included in the classification without a mask). The model that has been made has been able to work properly.

There is a difference in processing time for images without face images and there are face images. The time required for the system to process information without a facial image in it is an average time of 0.0943 seconds per frame. The longer time experienced by the system when there is one face image, which is 0.3471 seconds per frame. This is natural because the program does some pre-processing in order to obtain an accurate classification value. An example is matching the dimensions of the image taken through the camera, then image processing which is similar to image processing before the model is created.

\section{Conclusions}

In this article, we have studied the implementation of using three base deep-learning models using the MobileNetV2, ResNet50V2, and Xception methods to be fine-tuned in the case of facial image recognition using masks. We get the results that the use of ResNet50V2 and Xception has a good level of data validation accuracy when compared to MobileNetV2 which results in overfitting on the same research parameters. In addition, we get the result that MobileNetV2 tends to be faster when compared to ResNet50V2 and Xception.

\section{Declaration of Interest}

The author declares that no conflict of interest regarding financial or personal relationships affects the work reported in this article.

\section{References}

[1] T. Greenhalgh, M. B. Schmid, T. Czypionka, D. Bassler, and L. Gruer, "Face masks for the public during the covid-19 crisis," BMJ, vol. 369, no. April, pp. 1-4, 2020, doi: 10.1136/bmj.m1435.

[2] P. Bahl, C. Doolan, C. de Silva, A. A. Chughtai, L. Bourouiba, and C. R. MacIntyre, "Airborne or Droplet Precautions for Health Workers Treating Coronavirus Disease 2019?,” J. Infect. Dis., no. Xx Xxxx, pp. 1-8, 2020, doi: 10.1093/infdis/jiaa189.

[3] M. Otoom, N. Otoum, M. A. Alzubaidi, Y. Etoom, and R. Banihani, "An IoT-based framework for early identification and monitoring of COVID-19 cases," Biomed. Signal Process. Control, vol. 62, no. July, p. 102149, 2020, doi: 10.1016/j.bspc.2020.102149.

[4] A. Kumar, K. Sharma, H. Singh, S. G. Naugriya, S. S. Gill, and R. Buyya, "A drone-based networked system and methods for combating coronavirus disease (COVID-19) pandemic," Futur. Gener. Comput. Syst., vol. 115, pp. 1-19, 2021, doi: 10.1016/j.future.2020.08.046.

[5] S. Tuli, S. Tuli, R. Tuli, and S. S. Gill, "Predicting the growth and trend of COVID-19 pandemic 
using machine learning and cloud computing," Internet of Things, vol. 11, p. 100222, 2020, doi: 10.1016/j.iot.2020.100222.

[6] J. Wang, L. Pan, S. Tang, J. S. Ji, and X. Shi, "Mask use during COVID-19: A risk adjusted strategy," Environ. Pollut., vol. 266, no. 7, p. 115099, 2020, doi: 10.1016/j.envpol.2020.115099.

[7] Chandrikadeb7, "Face Mask Detection." [Online]. Available: https://github.com/chandrikadeb7/Face-Mask-Detection.

[8] Zhangyang, "Real World Masked Face Dataset." [Online]. Available: https://github.com/Xzhangyang/Real-World-Masked-Face-Dataset.

[9] M. Sandler, A. Howard, M. Zhu, A. Zhmoginov, and L. C. Chen, "MobileNetV2: Inverted Residuals and Linear Bottlenecks," Proc. IEEE Comput. Soc. Conf. Comput. Vis. Pattern Recognit., pp. 4510-4520, 2018, doi: 10.1109/CVPR.2018.00474.

[10] K. He, X. Zhang, S. Ren, and J. Sun, "Identity mappings in deep residual networks," Lect. Notes Comput. Sci. (including Subser. Lect. Notes Artif. Intell. Lect. Notes Bioinformatics), vol. 9908 LNCS, pp. 630-645, 2016, doi: 10.1007/978-3-319-46493-0_38. 